\title{
A Partial Reinforcement Extinction Effect Despite Equal Rates of Reinforcement During Pavlovian Conditioning
}

\author{
Mark Haselgrove \\ Cardiff University
}

\author{
Aydan Aydin \\ University of Mersin
}

\author{
John M. Pearce \\ Cardiff University
}

\begin{abstract}
In 4 experiments rats received appetitive Pavlovian conditioning followed by extinction. Food accompanied every trial with the conditioned stimulus (CS) for the continuously reinforced groups and only half of the trials for the partially reinforced groups. In contrast to previous experiments that have compared the effects of partial and continuous reinforcement, the rate at which food was delivered during the CS was the same for both groups. The strength of the conditioned response during extinction weakened more rapidly in the continuously than in the partially reinforced groups. The results demonstrate that the partial reinforcement extinction effect is a consequence of the nonreinforced trials with the CS, rather than the rate at which the unconditioned stimulus is delivered during the CS.
\end{abstract}

For many years the partial reinforcement extinction effect (PREE) was a mainstay of research in the field of animal learning and behavior. Within the context of instrumental conditioning, the PREE can be defined as a resistance to the detrimental effects of extinction following trials in which responding is intermittently rather than consistently paired with reinforcement (for a review see Mackintosh, 1974). So widespread was the PREE in studies of instrumental conditioning - and such was the paucity of evidence for the effect within Pavlovian conditioning - that for some time the PREE was thought to differentiate these two forms of conditioning (Kimble, 1961). However, the possibility that the PREE contradicted the single-process rule of learning (Guthrie, 1935; Hull, 1943) has not stood the test of time. Many studies of Pavlovian conditioning have now demonstrated that a conditioned stimulus (CS) that is intermittently paired with an unconditioned stimulus (US) will show a resistance to extinction relative to a CS that is consistently paired with a US (e.g., Fitzgerald, 1963; Fitzgerald, Vardaris, \& Teyler, 1966; Gibbon, Farrell, Locurto, Duncan, \& Terrace, 1980; Gibbs, Latham, \& Gormezano, 1978; Pearce, Redhead, \& Aydin, 1997; Rescorla, 1999b; Slivka \& Bitterman, 1966). This has not prevented the PREE from continuing to be a thorn in the side of theories of learning, however. From Thorndike (1911) onward (e.g., Rescorla \& Wagner, 1972), theo-

Mark Haselgrove and John M. Pearce, School of Psychology, Cardiff University, Cardiff, Wales, United Kingdom; Aydan Aydin, Department of Psychology, University of Mersin, Mersin, Turkey.

This research was supported by a grant from the Biotechnology and Biological Sciences Research Council of the United Kingdom.

Correspondence concerning this article should be addressed to Mark Haselgrove or John M. Pearce, School of Psychology, Cardiff University, Cardiff, Wales CF10 3YG, United Kingdom. E-mail: haselgrovem@ cardiff.ac.uk or pearcejm@ cardiff.ac.uk ries of learning have been based on the assumption that the strength of the association between a stimulus and a response, or between a CS and a US, determines the strength of conditioned responding. The nonreinforced trials that occur with partial reinforcement are predicted to result in a weaker association than when a continuous reinforcement schedule is used, and it therefore follows that extinction will progress more rapidly after partial than after continuous reinforcement.

To explain the PREE in instrumental conditioning, several theorists have proposed that nonreinforced trials have an effect in addition to weakening associative strength. In particular, such trials are assumed to generate an internal state of frustration (e.g., Amsel, 1958, 1992) or a memory trace of nonreinforcement (e.g., Capaldi, 1967). On the one hand, when extinction takes place after training with a continuous reinforcement schedule, the introduction of this state or trace for the first time will change the context in which the response occurs and thereby facilitate extinction through a generalization decrement (see Mackintosh, 1974, pp. $440-443)$. On the other hand, training with a partial reinforcement schedule provides an opportunity for responses to be reinforced in the context of nonreinforcement and thereby minimizes its disruptive effect during extinction.

Pearce et al. (1997) have used a similar line of reasoning to explain the effects of partial reinforcement on the extinction of Pavlovian conditioned responding. According to this view, partial reinforcement will allow the association between the CS and US to develop within the context of the aftereffects of nonreinforced trials and therefore reduce the generalization decrement engendered by these aftereffects during extinction. It should be evident from this perspective that the presence of nonreinforced trials with the CS during acquisition is crucial for a successful demonstration of the PREE in Pavlovian conditioning. Gallistel and Gibbon (2000, 2002), however, have argued that another property of partial reinforcement is responsible for the PREE in Pavlovian 
conditioning. According to Gallistel and Gibbon, extinction after Pavlovian conditioning is the consequence of a decision to stop responding. This decision is based on the value of two variables: $I \hat{R} I C S$ - the average duration of exposure to the CS between successive presentations of the US during conditioning and ICS no $R$ - the duration of exposure to the CS since the US was last delivered during the CS (Gallistel \& Gibbon, 2000, pp. 306, 343). When the ratio of these two variables ( $\hat{I}_{C S}$ no R/IR̂RCS) exceeds a decision threshold, the animal stops responding and extinction can be thought to have occurred. As a concrete example of the application of this theory to the PREE, consider two groups of rats undergoing appetitive Pavlovian conditioning. Both groups receive the same number of presentations of a 10 -s tone within a session; food is presented after the CS for half the trials for a partially reinforced group and all the trials for a continuously reinforced group. In these circumstances, the value of $I \hat{R} I_{C S}$ will be $10 \mathrm{~s}$ for the continuously reinforced group and $20 \mathrm{~s}$ for the partially reinforced group. Therefore, the total duration of nonreinforced exposure to the CS that must accumulate before the value of the decision variable exceeds the decision threshold will be less for the former group than the latter. That is, conditioned responding will cease sooner during extinction following continuous reinforcement than following partial reinforcement. A prediction that can be derived from this account of extinction is that partial reinforcement will not affect the number of USs that must be omitted before the subject decides to stop responding. In support of this prediction, Gibbon et al. (1980) showed that the PREE was not evident when examined in terms of the omission of expected USs.

Unlike associative theories, the account of extinction offered by Gallistel and Gibbon $(2000,2002)$ does not need to appeal to any additional processes to explain the PREE. For this reason alone, the theory merits serious attention. Accordingly, the four experiments reported in this article examine whether the rate at which the US is presented during the CS is responsible for determining whether a PREE will be observed. In each experiment, at least two groups of rats received appetitive Pavlovian conditioning with schedules that ensured they received the same rate of food delivery during the CS. This was achieved either by manipulating the number of USs delivered during each trial or by manipulating the duration of the trials. For one group, but not the other, the schedule also contained nonreinforced trials with the CS. If the proposals of Gallistel and Gibbon are correct, then the performance of the two groups during extinction will be identical. However, if the PREE depends on the presence of nonreinforced trials during acquisition, then a difference between the groups should be observed during extinction.

\section{Experiment 1}

In Experiment 1, two groups of rats received the same duration of exposure to a CS during conditioning and also the same number of presentations of the US. Every trial with the CS was accompanied by food for the continuously reinforced group, whereas for the partially reinforced group food was delivered on half of the trials with the CS. The duration of each trial was $10 \mathrm{~s}$ for both groups. To equate the groups in terms of the rate of reinforcement during the CS, food was presented once on every trial to the continuously reinforced group and twice on every reinforced trial to the partially reinforced group - once after $5 \mathrm{~s}$ and once at the end of the trial.
The continuously reinforced group also received food at these intervals, but on different trials. Experiments conducted in our laboratory (Haselgrove \& Pearce, 2003) have shown that the latter schedule is effective in producing conditioned responding. In addition, it has the advantage of ensuring that both groups in the current experiment are reinforced both at the termination of the CS and $5 \mathrm{~s}$ earlier. The design of the conditioning stage is shown in Figure 1. On the completion of conditioning, both groups received nonreinforced trials with the 10-s CS. If the PREE is a consequence of presenting nonreinforced trials with the CS during conditioning then, in contrast to the prediction of the theory of Gallistel and Gibbon (2000), extinction should progress more rapidly in Group Continuous than in Group Partial.

\section{Method}

Subjects. The subjects were 16 experimentally naive, male, hooded Lister rats (Rattus norvegicus) supplied by Harlan Olac (Bicester, Oxon, England). Prior to the start of the experiment, at an age of approximately 3 months, they were gradually reduced to $80 \%$ of their free-feeding weights. They were maintained at these weights throughout the experiment by being fed a restricted amount after each experimental session. The rats were housed in pairs in a light-proof room in which the lights were on for $14.5 \mathrm{hr} /$ day. The subjects were tested at the same time on successive days during the period when the lights were on in their holding room.

Apparatus. Eight conditioning chambers $(24.5 \times 23.0 \times 20.0 \mathrm{~cm})$ were housed in separate light- and sound-attenuating chests. Exhaust fans in each chest provided a background masking noise of $72 \mathrm{~dB}$ (C scale). Three walls of each chamber were constructed from aluminum, one side was clear acrylic, and the ceiling was translucent white acrylic. A $5-\Omega$ speaker located on the center of the ceiling delivered a $10-\mathrm{Hz}$ click at an intensity of $83 \mathrm{~dB}(\mathrm{C}$ scale). There was a $5.0 \times 6.0 \mathrm{~cm}$ recessed food magazine in the front wall into which $45-\mathrm{mg}$ food pellets (traditional formula, P. J. Noyes, Lancaster, NH) could be delivered, its base was located $0.5 \mathrm{~cm}$ above the grid floor. Subjects were required to push open a clear acrylic flap in order to gain access to the magazine. Three pairs of photodiode sensors were set into a rectangular frame $1-\mathrm{cm}$ deep that surrounded the magazine entrance in such a manner that horizontal beams, $5 \mathrm{~mm}$ in front of the closed magazine flap, were located 10, 20, and $30 \mathrm{~mm}$ above the grid floor. Appropriate circuitry permitted an Archimedes microcomputer (Acorn Computers Ltd., Cambridge, England), which was programmed in Arachnid (Paul Fray Ltd., Cambridge, England) to record the interruption of these beams and to control the experimental events.

Procedure. All rats initially received a single session of magazine training. During this 40-min session, one food pellet was delivered at regular 1-min intervals. For this session only, the acrylic flap of the magazine was taped open. The subjects were then divided into two groups of equal size. In each of 12 sessions of conditioning there were 20 presentations of a 10-s clicker. The interval between the end of one
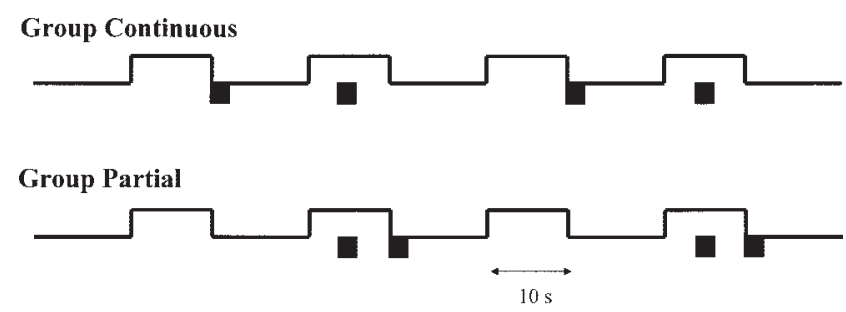

Figure 1. Procedure and design of Experiment 1. Time is indicated left to right. Open boxes represent conditioned stimulus presentations. Black boxes represent unconditioned stimulus presentations. 
presentation of the clicker and the start of the next trial, the intertrial interval (ITI), had a mean duration of $120 \mathrm{~s}$ (range $=90-150 \mathrm{~s}$ ). For Group Continuous, the termination of half of the conditioning trials was paired with the delivery of a single food pellet. For the remaining trials, a single food pellet was delivered $5 \mathrm{~s}$ after the onset of the clicker. The sequence of these trials was random with the constraint that no more than two trials of the same type could occur in succession. Half of the presentations of the CS for Group Partial in each session were not paired with food. These trials occurred at the same point within the session as the trials with food presented at the end of the clicker in Group Continuous. On each of the remaining trials, two food pellets were delivered. The first occurred $5 \mathrm{~s}$ after the onset of the clicker and the second immediately after the end of the clicker. Following the final session of conditioning, the rats received 2 sessions of extinction. In each of these sessions there were 18 nonreinforced trials with a 10-s clicker. The ITI for these trials was again $120 \mathrm{~s}$ (range $=90-150 \mathrm{~s})$.

The measure of conditioning was magazine activity that was deemed to have taken place whenever the infrared beams in front of the magazine were broken. The duration of magazine activity was recorded for $10 \mathrm{~s}$ prior to every trial in both stages of the experiment. The duration of this activity was also recorded in the presence of the CS on nonreinforced trials in the partial group and on trials where food was delivered at the termination of the CS in Group Continuous. Magazine activity was also recorded during each extinction trial. A difference score was calculated by subtracting the duration of magazine activity during the pre-CS period from the duration of magazine activity during the CS period.

\section{Results and Discussion}

A Type I error rate of $p<.05$ was adopted for all the statistical tests. One subject in Group Partial failed to engage in any magazine activity and was discarded from the experiment.

The mean difference scores for Groups Partial and Continuous throughout the 12 sessions of conditioning can be seen in the left panel of Figure 2. There is an indication that conditioning was initially more effective in Group Continuous than in Group Partial. However, this observation was not supported by statistical analysis. A two-way analysis of variance (ANOVA) of individual difference scores for the two groups for each of the 12 sessions of conditioning revealed a significant effect of session, $F(11,143)=$
76.50, but no effect of group $(F<1)$, and no Group $\times$ Session interaction, $F(11,143)=1.42$. The mean durations of magazine activity for Groups Partial and Continuous across the 12 sessions of conditioning during the 10-s intervals prior to the conditioning trials were 1.21 and $1.65 \mathrm{~s}$, respectively. This difference was not significant, $t(13)=2.12$.

The group mean difference scores are shown, in two-trial blocks, for the two sessions of extinction in the right panel of Figure 2. In both extinction sessions the mean duration of magazine activity declined more rapidly in Group Continuous than in Group Partial. A two-way ANOVA of difference scores, in twotrial blocks, revealed a significant effect of group, $F(1,13)=8.72$, and of two-trial block, $F(17,221)=8.11$, but no Group $\times$ Block interaction $(F<1)$. The mean durations of magazine activity during the 10-s periods prior to the onset of the clicker for Groups Partial and Continuous across the two sessions of extinction were 0.36 and $0.22 \mathrm{~s}$, respectively. The difference between these means was not significant, $t(13)=1.67$.

Gallistel and Gibbon (2000) stated that extinction occurs when subjects make a decision to stop responding. To identify when this decision was made in the present experiment, we used the extinction criterion adopted by Gallistel and Gibbon (p. 306) of a decline to $50 \%$ of the preextinction rate of responding. Extinction was therefore deemed to be effective when the difference score was, for two consecutive two-trial blocks, less than half that measured during the final session of conditioning. The number of trial blocks that elapsed before this criterion was met was 13.29 for Group Partial and 8.25 for Group Continuous. This difference was significant, $t(13)=2.50$.

Given that both groups received the same rate of reinforcement during the CS, the theory of Gallistel and Gibbon (2000) predicts that they should perform similarly during the extinction stage of the experiment. Thus the greater resistance to extinction that was observed in Group Partial than in Group Continuous poses a challenge to the explanation for the PREE that can be derived from this theory. In contrast, the results are consistent with the suggestion that the PREE is a consequence of the nonreinforced trials that
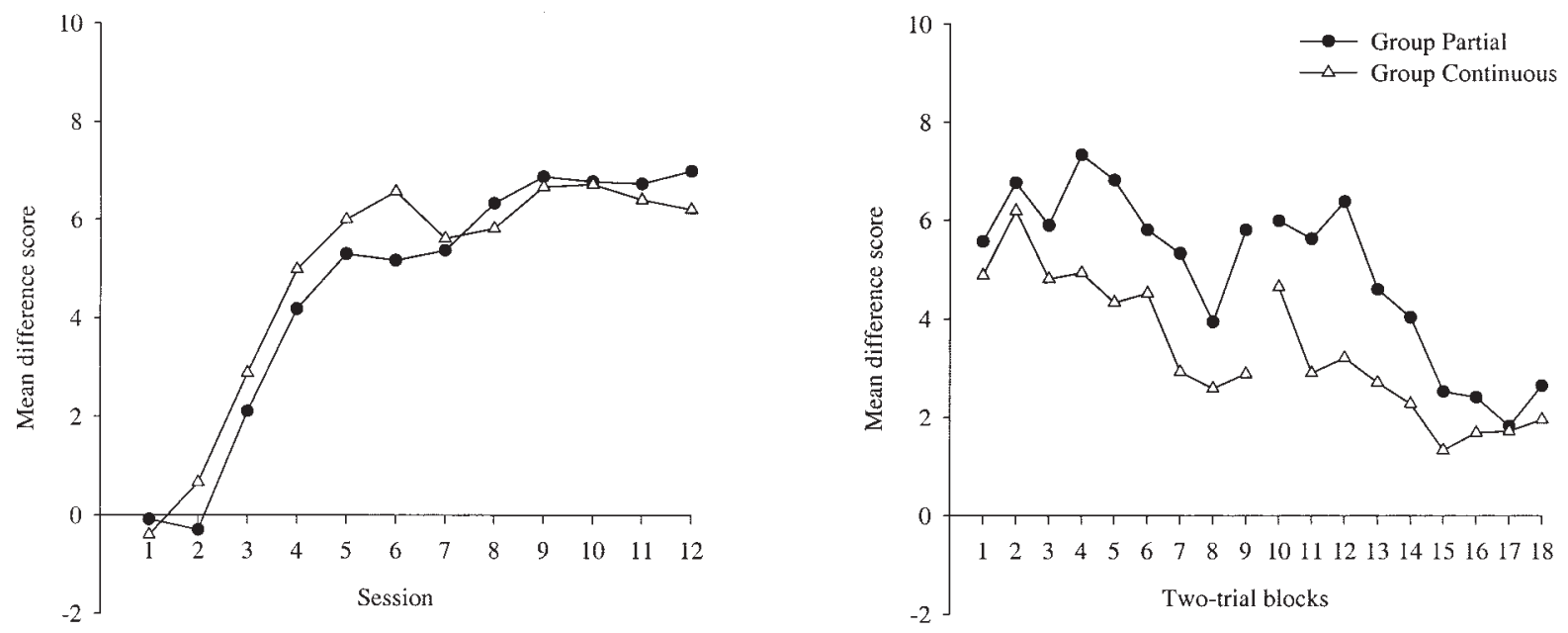

Figure 2. The mean difference scores for Group Partial and Group Continuous across the 12 sessions of conditioning (left panel) and across the 2 sessions of extinction (right panel) of Experiment 1. 
occur intermittently throughout conditioning (Pearce et al., 1997). There is, however, another way in which the results can be explained. The groups differed not only in their reinforcement schedules but also with respect to the frequency with which food was delivered within reinforced trials. Reinforcement for Group Continuous consisted of a single food pellet on a trial, whereas for Group Partial it consisted of a single food pellet followed $5 \mathrm{~s}$ later by another pellet. It is conceivable that it was this difference, rather than the different reinforcement schedules, that was responsible for the slower rate of extinction in the partial than the continuous group. A measure of support for this suggestion can be found in experiments which have shown that extinction progresses more slowly after Pavlovian conditioning has taken place with a large rather than a small US. For example, Wagner, Siegel, Thomas and Ellison (1964) reported that conditioned salivation extinguished more slowly if the CS had been paired with six pellets of food on each trial instead of one pellet. If two food pellets separated by a short interval constitute a larger US than one pellet, then whatever explanation is deemed appropriate for the results reported by Wagner et al. may also apply to the present results. This possibility was tested in Experiment 2.

\section{Experiment 2}

There were four groups in this experiment, two of which received the same treatment during conditioning as Groups Continuous and Partial from Experiment 1 (now labeled Group Continuous-1 and Group Partial-2, respectively). In addition, two further groups were included that were again conditioned with either a continuous or partial reinforcement schedule. For Group Continuous-2, the CS was paired with two food pellets on each trial, one delivered $5 \mathrm{~s}$ after the start of the CS and another $5 \mathrm{~s}$ later. For Group Partial-1, half of the conditioning trials were nonreinforced, and for the remainder a single pellet of food was presented either $5 \mathrm{~s}$ after the onset of the clicker or at its termination. The design of the conditioning stage for Experiment 2 is shown in Figure 3 .
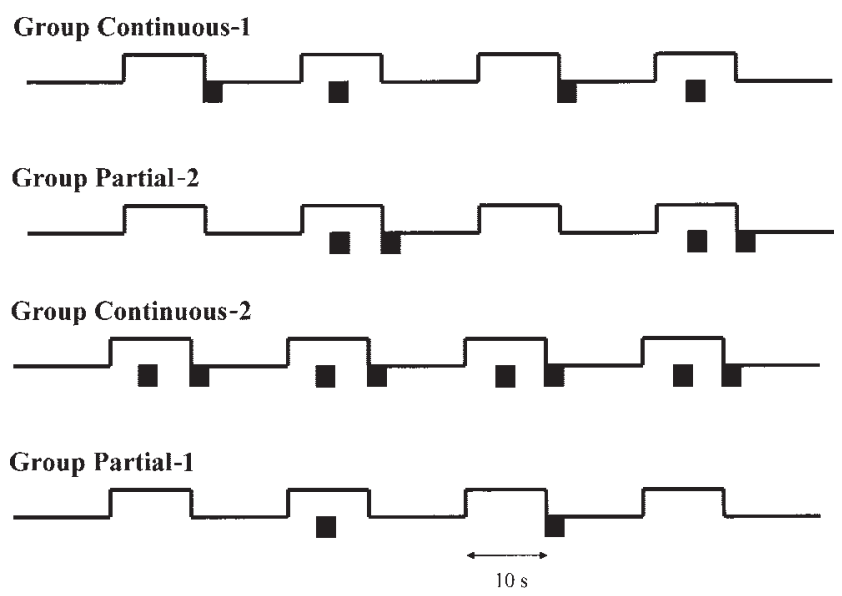

Figure 3. Procedure and design of Experiment 2. Time is indicated left to right. Open boxes represent conditioned stimulus presentations. Black boxes represent unconditioned stimulus presentations.
If presenting food twice during each reinforced trial was responsible for the subsequent slow rate at which responding weakened during extinction with Group Partial of Experiment 1, then extinction should be more rapid in Group Continuous-1 than in Group Partial-2. In addition, extinction should be more rapid in Group Partial-1 than in Group Continuous-2. Alternatively, if the presence of nonreinforced trials during conditioning determines the rate of extinction, then the strength of conditioned responding should decline more rapidly in both of the continuously reinforced groups than in either of the partially reinforced groups. Finally, the account of extinction provided by the theory of Gallistel and Gibbon (2000) leads to the prediction that extinction will progress most rapidly in Group Continuous-2, somewhat more slowly in Groups Partial-2 and Continuous-1 (which will not differ from each other), and most slowly in Group Partial-1. The justification for these predictions can be appreciated by an examination of Figure 3. According to the theory of Gallistel and Gibbon, extinction will progress most rapidly in a group whose CS has the highest rate of reinforcement. Each group in the current experiment receives the same duration of exposure to the CS. Therefore the rate of extinction should be directly related to the number of USs delivered during conditioning, with higher numbers of USs resulting in faster extinction.

\section{Method}

Subjects and apparatus. The subjects were 32 male rats from the same stock and maintained in the same manner as for Experiment 1. The apparatus was the same as for Experiment 1.

Procedure. All subjects were first magazine trained in the same manner as Experiment 1. They were then divided into four groups of equal size, and given 13 sessions of conditioning in each of which there were 20 presentations of a 10-s clicker CS. For Groups Partial-2 and Continuous-1 the procedure was the same, respectively, as for Groups Partial and Continuous from Experiment 1. For Group Continuous-2, two food pellets were delivered on every trial in the same manner as the reinforced trials for Group Partial-2. For Group Partial-1, food was presented on only half the trials, either $5 \mathrm{~s}$ after the onset of the CS or immediately after its termination. Following the final session of conditioning, the rats received two sessions of extinction. The first 8 trials of the first extinction session proceeded as in the previous conditioning session. The remainder of this session comprised 14 nonreinforced trials with the 10-s clicker, which were then repeated for the second extinction session. The mean ITI throughout the experiment, for all groups, was $120 \mathrm{~s}$ (range $=90-150 \mathrm{~s}$ ).

Food was always presented $5 \mathrm{~s}$ after the start of each trial for Group Continuous-2, which made it impossible to measure performance throughout the presentation of the clicker without contamination from responding brought about by the delivery of this food pellet. To circumvent this problem, magazine activity during conditioning was measured only during the first $5 \mathrm{~s}$ of the clicker in each group. For extinction, magazine activity was measured throughout each trial with the clicker. Procedural details that have been omitted were the same as for Experiment 1.

\section{Results and Discussion}

The mean difference scores for the four groups throughout the 13 sessions of conditioning can be seen in the left panel of Figure 4. There were no statistically significant differences among the groups. A three-way ANOVA of individual difference scores with the factors of number of food pellets delivered on a trial, conditioning schedule, and session revealed no effect of number of 

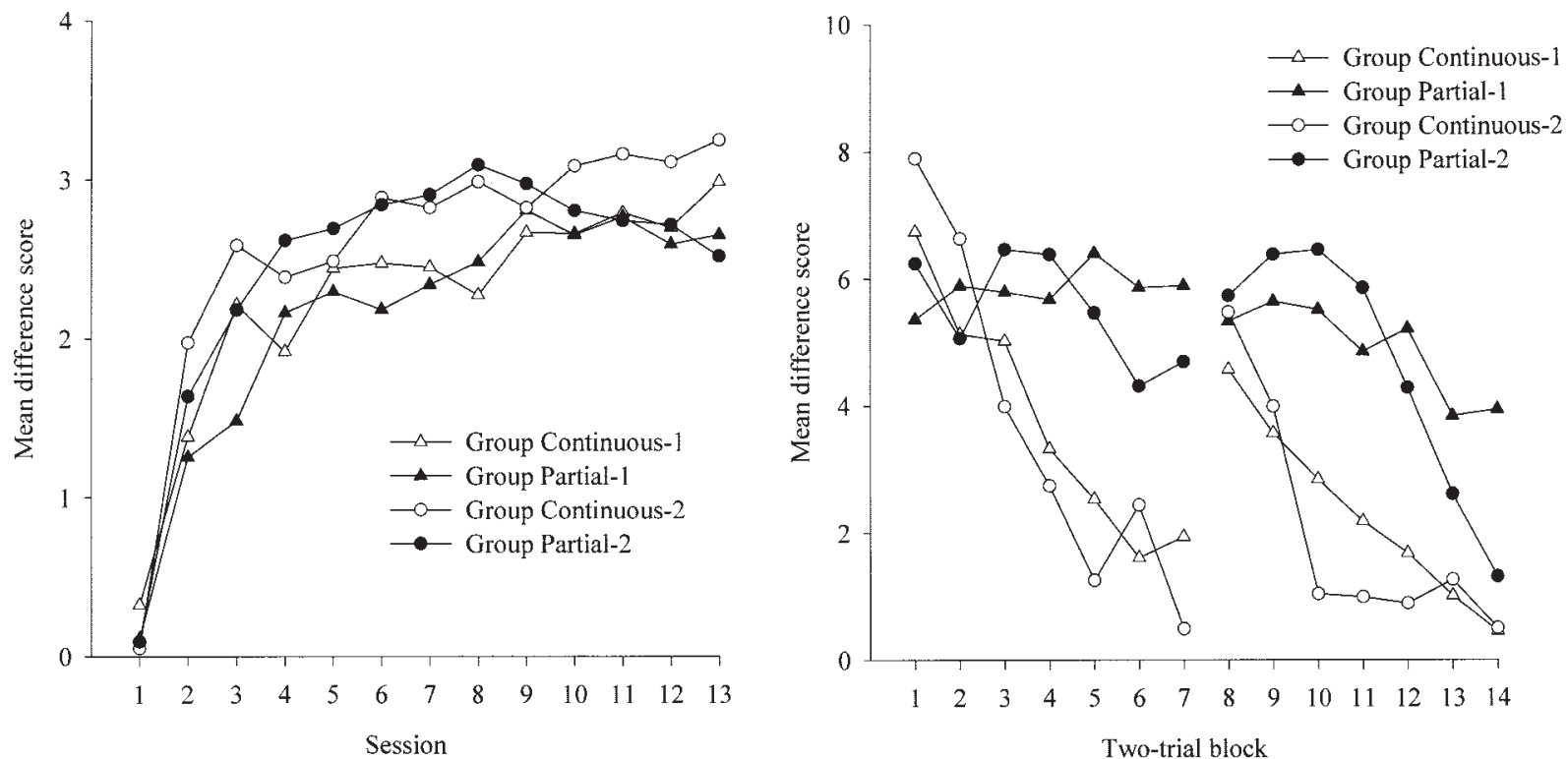

Figure 4. The mean difference scores for Groups Continuous-1, Partial-1, Continuous-2, and Partial-2 across the 13 sessions of conditioning (left panel) and across the 2 sessions of extinction (right panel) of Experiment 2 .

food pellets, $F(1,28)=3.33$, and no effect of schedule $(F<1)$. There was however a significant effect of session, $F(12,36)=$ 60.14. The Number of Food Pellets $\times$ Schedule interaction was not significant $(F<1)$, and neither was the Number of Food Pellets $\times$ Session interaction, or the Schedule $\times$ Session interaction, $F \mathrm{~s}(12,336)<1.56$. The three-way interaction also fell short of significance $(F<1)$. The mean durations of magazine activity during the 5-s period prior to the onset of the CS for all conditioning sessions were $0.60 \mathrm{~s}$ for Group Continuous-1, $0.57 \mathrm{~s}$ for Group Partial-1, $0.69 \mathrm{~s}$ for Group Continuous-2, and $0.69 \mathrm{~s}$ for Group Partial-2. A two-way ANOVA of mean durations of magazine activity revealed no effect of number of reinforcers, no effect of schedule, and no interaction, $F \mathrm{~s}(1,28)<3.35$.

The group mean difference scores are shown, in two-trial blocks, for the two sessions of extinction in the right panel of Figure 4. In both sessions the strength of conditioned responding declined more rapidly in the two continuously reinforced groups than in either of the two partially reinforced groups. A three-way ANOVA of difference scores, in two-trial blocks, revealed no effect of number of food pellets $(F<1)$, but significant effects of schedule, $F(1,28)=16.65$, and trial block, $F(13,364)=25.38$. The Number of Food Pellets $\times$ Schedule interaction was not significant $(F<1)$, but the Number of Food Pellets $\times$ Trial Block interaction and the Schedule $\times$ Trial Block interactions were both significant, $F \mathrm{~s}(13,354)>1.78$. The three-way interaction was also significant, $F(13,364)=2.39$. Subsequent tests of simple main effects revealed a significant difference between Groups Partial-1 and Continuous- 1 on Trial Blocks 4 to 7 and 10 to 14, $F \mathrm{~s}(1,392)>4.62$; and significant differences between Groups Partial-2 and Continuous-2 on Trial Blocks 3, 4, 5, 7 and 9 to 12, $F_{\mathrm{s}}(1,392)>4.74$. On Trial Block 14 there was a significant difference between Groups Partial-1 and Partial-2, $F(1,392)=$ 5.77 .
The mean durations of magazine activity during the 5-s periods prior to the onset of the clicker, for all the extinction trials, were $0.15 \mathrm{~s}$ for Group Continuous-1, $0.18 \mathrm{~s}$ for Group Partial-1, $0.16 \mathrm{~s}$ for Group Continuous-2, and $0.19 \mathrm{~s}$ for Group Partial-2. The differences among these means were not significant $\left(F_{\mathrm{S}}<1\right)$.

The number of two-trial blocks that elapsed before the extinction criterion was met were 6.50 for Group Continuous-1, 13.88 for Group Partial-1, 7.00 for Group Continuous-2, and 11.13 for Group Partial-2. A two-way ANOVA of criterion scores revealed a significant effect of schedule, $F(1,28)=25.21$, but no effect of number of food pellets $(F<1)$, and no Number of Food Pellets $\times$ Schedule interaction, $F(1,28)=2.01$.

In keeping with the results from Experiment 1, the present results again show the importance of nonreinforced trials during training for the PREE in Pavlovian conditioning. At the same time, they lend no support to the explanation for this effect put forward by Gallistel and Gibbon (2000). It follows from this theory that extinction should have progressed at the same rate in Group Partial-2 and Continuous-1, whereas the duration of magazine activity during the test trials was generally longer in the partially reinforced group. The possibility was raised earlier that the difference between the results for these two groups might have occurred because the manner in which the US was delivered made it a more effective reinforcer for Group Partial-2 than for Group Continuous-1. If this were the case, then extinction should have progressed more slowly in Group Continuous-2 than in Group Partial-1. In fact, the opposite outcome was observed.

\section{Experiment 3}

If the PREE that was demonstrated in Experiment 1 was not a consequence of two food pellets presented in close succession being a more effective reinforcer than when they are presented 
separately, then it may be possible to explain the results by assuming that these different ways of presenting the US had the opposite effect. That is, delivering one pellet shortly after the other may have rendered food a less effective reinforcer than when the pellets were presented individually. For instance, it is possible that the effectiveness of the second food pellet to act as a reinforcer was diminished by the presentation of an identical food pellet $5 \mathrm{~s}$ earlier. Carew, Pinsker, and Kandel (1972) and Pfautz (1980) have both reported that the capacity for a US to evoke an unconditioned response can be diminished if it is preceded by the same US. Furthermore, Terry (1976) has shown how unsignaled pretrial US exposure renders that US less effective as a reinforcer when it is subsequently paired with a CS in Pavlovian conditioning. An implication of these findings is that presenting two food pellets with an interval of $5 \mathrm{~s}$ between them might render the second food pellet ineffective as a US for Pavlovian conditioning. Once this possibility is acknowledged, then it follows that the rate at which the US was delivered during Group Partial of Experiment 1 was effectively less than for Group Continuous, and the theory of Gallistel and Gibbon (2000) predicts that a PREE should be observed.

The purpose of Experiment 3 was to determine whether it is possible to demonstrate a PREE in Pavlovian conditioning when the rate of delivery of the US during the CS is the same for the partial and continuous schedules and when the interval between successive presentations of the US is also the same. To this end, Group Partial-10 received conditioning with a 10 -s CS that was followed immediately by food on half the trials. In order to reproduce with continuous reinforcement the same rate of food delivery during the CS that was created by this intermittent schedule, the duration of the CS was extended from 10 to $20 \mathrm{~s}$ for Group Continuous-20. If food was delivered at the end of each of these trials, then it is possible that inhibition of delay (Pavlov, 1927) would weaken responding at the start of the CS to a greater extent than for Group Partial-10 and would make it difficult to interpret any difference between the groups that might be observed during extinction. Accordingly, for Group Continuous-20, food was presented $10 \mathrm{~s}$ after the start of the CS on half the trials and at the end of the CS on the remaining trials (see Figure 5). It was hoped that this schedule would result in similar performance by both groups during the initial $10 \mathrm{~s}$ of each trial.

The treatments just described ensured that the two groups received the same rate of reinforcement during the CS and, accord-
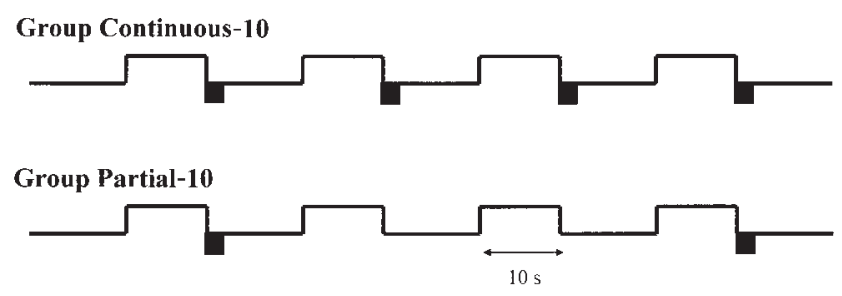

Group Continuous-20

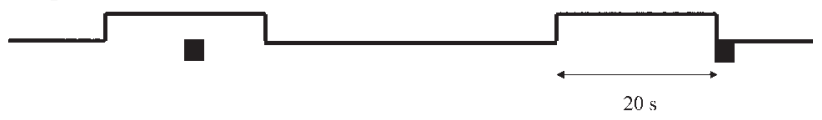

Figure 5. Procedure and design of Experiment 3. Time is indicated left to right. Open boxes represent conditioned stimulus presentations. Black boxes represent unconditioned stimulus presentations. ing to the theory of Gallistel and Gibbon $(2000,2002)$, they should perform similarly during extinction. In addition, both groups received only one pellet of food on each reinforced trial, and the trials were ordered and spaced in such a fashion that the delivery of food for these groups occurred at the same point within a session. The two groups were thus matched not only on their rate of reinforcement during the CS but also on their distribution of reinforcement during conditioning. The effectiveness of food as a reinforcer should therefore be the same for the two groups, which means it would not be reasonable to explain any difference between them during testing in terms of the explanation that was offered for Experiment 2. On the other hand, if prior experience of a partial reinforcement schedule should weaken the effects of extinction, then conditioned responding on the test trials will be greater in Group Partial-10 than in Group Continuous-20.

A third group, Group Continuous-10, that was included in the experiment received the same sequence and duration of trials as Group Partial-10, but food was presented at the end of every trial. The rate of reinforcement during the CS for this group was greater than for Group Continuous-20, so that according to the theory of Gallistel and Gibbon (2000), the effects of extinction should become evident more rapidly for the group trained with the CS of shorter duration. Once again, a different prediction is made if the principal determinant of the effects of extinction is whether subjects have experienced partial or continuous reinforcement. On this basis, little difference between the groups would be expected. Of course, responding during the test trials should weaken more slowly in Group Partial-10 than Group Continuous-10.

Although the groups received different durations of the CS for conditioning, to simplify the interpretation of the results from the experiment they all received extinction test trials with a 20 -s CS We chose to use this value because the likely effect of increasing the duration of the CS for the extinction stage in Group Partial-10 would be to weaken responding through a generalization decrement (see Haselgrove \& Pearce, 2003). Accordingly, if the experiment should reveal a PREE with these groups, it will be difficult to attribute it to the change in duration of the CS in Group Partial-10.

\section{Method}

Subjects and apparatus. The subjects were 24 male rats from the same stock and maintained in the same manner as for Experiment 1. The apparatus was the same as for Experiment 1.

Procedure. All subjects were first magazine trained in the same manner as Experiment 1. The subjects were then divided into three groups of equal size. For Groups Continuous-10 and Partial-10, each of 13 sessions of conditioning contained 20 presentations of a 10-s clicker separated by an ITI of $120 \mathrm{~s}$ (range $=90-150 \mathrm{~s}$ ). For Group Continuous-10, the termination of each presentation of the clicker was paired with the delivery of a single food pellet. For Group Partial-10, the termination of a randomly selected half of the trials was paired with the delivery of a single food pellet. For Group Continuous-20, each conditioning session contained 10 presentations of a 20 -s clicker. The first trial began at the same point within the session as that for Group Partial-10. Subsequent trials were separated by an ITI of $240 \mathrm{~s}$ (range $=180-300 \mathrm{~s}$ ). For a randomly selected half of the trials food was presented $10 \mathrm{~s}$ after the start of the clicker, and for the remaining trials it was presented at the end of the clicker. The timing of events ensured that each food pellet was delivered at an identical point within a session for Group Partial-10 and Continous-20. To compare the 
performance of the three groups during conditioning, the duration of magazine activity was recorded for the first $10 \mathrm{~s}$ of each trial in each group.

The first 8 trials of the first extinction session for Groups Continuous-10 and Partial-10 and the first 4 trials for Group Continuous-20 proceeded as in the previous conditioning session. The remainder of this session comprised 14 nonreinforced trials with a 20-s clicker, which were repeated for the second extinction session. The duration of magazine activity was recorded for the entire duration of each trial during extinction. The mean ITI for the trials conducted in extinction for each group was $180 \mathrm{~s}$ (range $=$ $120-240$ s). Procedural details that have been omitted were the same as for Experiment 1.

\section{Results and Discussion}

The mean difference scores for the three groups for the 13 sessions of conditioning can be seen in the left panel of Figure 6. Acquisition progressed most rapidly in Group Continuous-10, at an intermediate rate in Group Continuous-20, and most slowly in Group Partial-10. These observations were supported by a twoway ANOVA of individual difference scores which revealed no effect of group, $F(2,21)=1.71$, but a significant effect of session, $F(12,252)=51.56$, and a significant Group $\times$ Session interaction, $F(24,252)=1.61$. Tests of simple main effects revealed that there were differences among the groups on Sessions 2, 3 and 8, Fs $(2$, 273) $>$ 3.36. Subsequent tests conducted according to the Newman-Keuls procedure (Kirk, 1968) revealed that on these three sessions, the mean difference score was greater in Group Continuous-10 than in Group Partial-10. On Session 3, the mean difference score was also significantly greater for Group Continuous-10 than Group Continuous-20, and on Session 8, the performance of Group Continuous-20 was superior to that of Group Partial-10. The fact that performance during the first half of the clicker was superior in Group Continuous-20 to Group Partial-10 demonstrates that the steps taken to prevent inhibition of delay from weakening responding during the first $10 \mathrm{~s}$ of the CS for Group Continuous-20 were effective. The mean durations of magazine activity during the 10 -s period prior to the onset of the clicker averaged across all the sessions of conditioning were $0.92 \mathrm{~s}$ for Group Continuous-10, $0.74 \mathrm{~s}$ for Group Partial-10, and $0.78 \mathrm{~s}$ for Group Partial-20. These differences were not significant $(F<1)$.

The group mean difference scores are shown, in two-trial blocks, for the two sessions of extinction in the right panel of Figure 6. In both extinction sessions the strength of conditioned responding declined more rapidly in the two continuously reinforced groups than in Group Partial-10. These observations were supported by a two-way ANOVA of difference scores, in two-trial blocks, which revealed no effect of group, $F(2,21)=1.26$, but a significant effect of trial block, $F(13,273)=42.95$, and a significant Group $\times$ Trial Block interaction, $F(26,273)=2.05$. Tests of simple effects revealed differences among the groups on Trial Blocks 7, 10, and 11, Fs $(2,294)>3.35$. Tests conducted according to the Newman-Keuls procedure revealed that on each of these trial blocks the mean difference score was smaller in Group Continuous-10 than in Group Partial-10. In addition, on Blocks 10 and 11, the mean difference score was smaller in Group Continuous-20 than in Group Partial-10. The mean durations of magazine activity during the 10 -s period prior to the onset of the clicker for all 14 two-trial blocks were $0.78 \mathrm{~s}$ for Group Continuous-10, 0.74 s for Group Partial-10, and $0.50 \mathrm{~s}$ for Group Continuous-20. The differences among these means were not significant $(F \mathrm{~s}<1)$.

The number of two-trial blocks that elapsed before the extinction criterion was met were 8.50 for Group Continuous-10, 12.00 for Group Partial-10, and 9.25 for Group Continuous-20. A oneway ANOVA of individual criterion scores revealed the difference among the groups was significant, $F(2,21)=6.52$. NewmanKeuls tests revealed that the mean criterion scores were greater in Group Partial-10 than in either Group Continuous-20 or Group Continuous-10.

The important results from the experiment concern Groups Partial-10 and Continuous-20. Both groups received the same duration of exposure to the CS in each conditioning session, and both groups received the US delivered at the same rate in the presence of the CS. According to the theory of Gallistel and Gibbon (2000), therefore, there should have been no difference
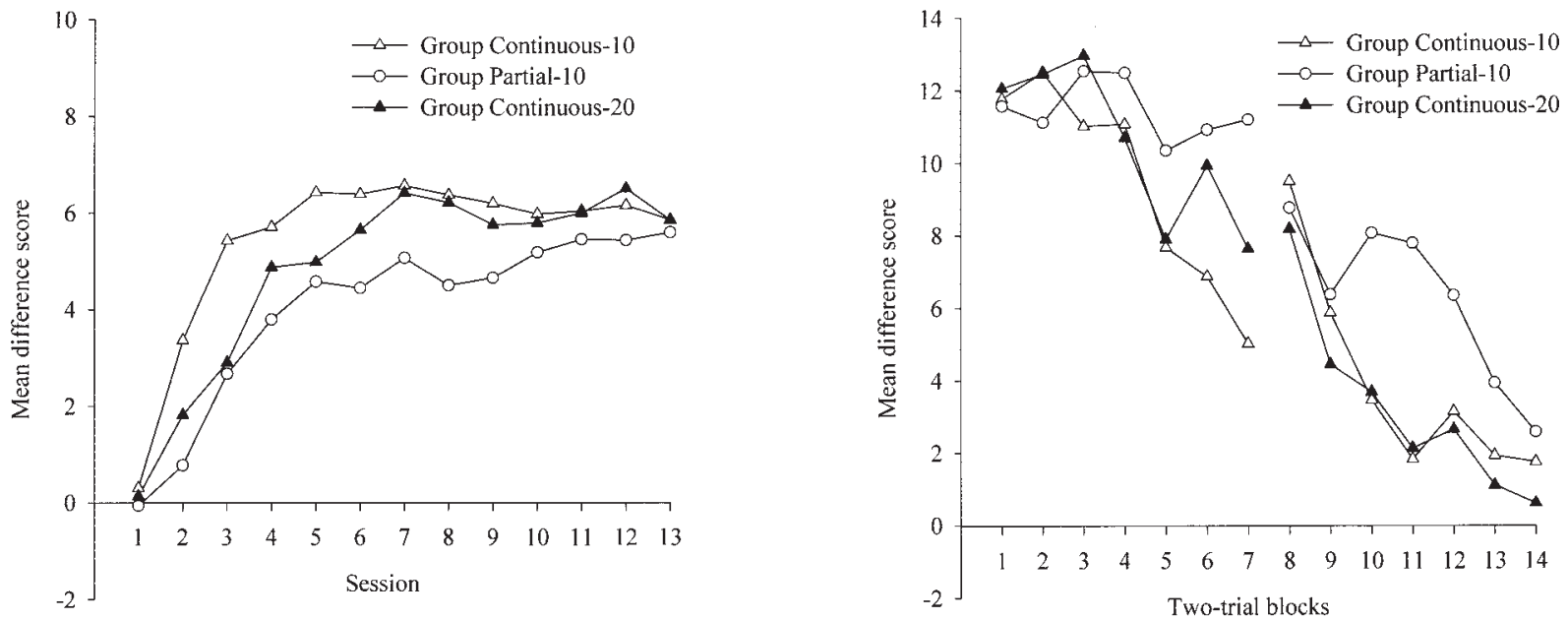

Figure 6. The mean difference scores for Groups Continuous-10, Partial-10, and Continuous-20 across the 13 sessions of conditioning (left panel) and across the 2 sessions of extinction (right panel) of Experiment 3. 
between these groups during the extinction trials, yet there was clear evidence of a PREE. In keeping with the results from the previous experiments, the present findings point to the nonreinforced trials with the CS during conditioning as being responsible for this effect. It might be argued that the slow rate of extinction that was observed in Group Partial-10, relative to Group Continuous-20, occurred because of the increase in the duration of the CS that took place between the conditioning and extinction stages of the experiment for the first of these groups. Although it is not possible to reject this explanation with complete confidence, there are at least two reasons for treating it lightly. First, several experiments have shown that instead of retarding the rate of extinction, an increase in the duration of the CS has the opposite effect of facilitating extinction (Haselgrove \& Pearce, 2003; Polin, 1959). Second, Group Continuous-10 also experienced an increase in the duration of the CS for the extinction phase, yet there was no hint that this manipulation strengthened responding relative to Group Continuous-20. Indeed, at the end of the first session there was an indication that the change in CS duration may have facilitated extinction.

The duration of the ITI was altered from conditioning to extinction for the three groups. This interval was increased from $120 \mathrm{~s}$ to $180 \mathrm{~s}$ for Group Partial-10 and Continuous-10, and decreased from $240 \mathrm{~s}$ to $180 \mathrm{~s}$ for Group Continuous-20. It is hard to predict how these changes might have affected the outcome of the experiment. Teichner (1952) reported that increasing or decreasing the ITI between conditioning and extinction facilitated the rate at which operant bar pressing extinguished, but we are not aware of a similar effect being reported for Pavlovian conditioning. It is worth noting, however, that there was rather little difference between the results from the two continuously reinforced groups, despite the different changes to the ITI that they received. On this basis, the most reasonable conclusion to draw would be that changing the ITI for the extinction phase had little impact on the outcome of the results for all three groups.

\section{Experiment 4}

On the reinforced trials of Experiment 3, food was always presented at the end of a 10-s CS for Group Partial-10, whereas it was presented at the midpoint or the end of a 20-s CS for Group Continuous-20. The reason for presenting food in the middle of some trials for Group Continuous-20 was to prevent the development of inhibition of delay. Although the results suggest this manipulation was successful, by adopting it we may have paved the way for our findings to be explained by the theory of Gallistel and Gibbon (2000). On those trials when food was delivered before the end of the CS, rats in Group Continuous-20 may have paid so much attention to the food that they ignored the fact that the CS was still present. This diversion of their attention would then result in the CS having an effective duration on these trials of 10 rather than $20 \mathrm{~s}$, and the rate of reinforcement during the CS would be less for Group Continuous-20 than for Group Partial-10. Once this possibility is acknowledged then it follows from the theory of Gallistel and Gibbon (2000) that extinction will progress more slowly in the partially than in the continuously reinforced group. In view of this possible explanation for the results from Experiment 3, another method was adopted in Experiment 4 for allowing the effects of partial reinforcement to be examined in groups that received identical rates of reinforcement during the CS.

Figure 7 shows the design for Experiment 4. For Group Continuous-10/20, the termination of each presentation of the CS, which had a duration of either 10 or $20 \mathrm{~s}$, was paired with the delivery of a single food pellet. The duration of the CS for Group Partial-10/5 was either 10 or $5 \mathrm{~s}$, and a randomly selected half of these presentations was followed by a single pellet of food. These values ensure that the rate of reinforcement during the CS was the same for both groups, and the theory of Gallistel and Gibbon (2000, 2002) predicts that they should perform similarly during extinction. To test this prediction, both groups received test trials with a 10-s CS. Because food was always presented at the end of the CS for both groups, the type of explanation just considered for Experiment 3 is not appropriate for the present study.

\section{Method}

Subjects and apparatus. The subjects were 16 male rats from the same stock and maintained in the same manner as for Experiment 1. The apparatus was the same as for Experiment 1 .

Procedure. All subjects were magazine trained in the same manner as Experiment 1. They were then divided into two groups of equal size. For Group Partial-10/5 each of 15 daily sessions of conditioning contained 8 trials with a 5 -s clicker and 8 trials with a 10 -s clicker. The termination of a randomly selected half of each type of trial was followed by the delivery of a single food pellet. The mean ITI was $240 \mathrm{~s}$ (range $=180-300 \mathrm{~s}$ ). For Group Continuous-10/20 every session contained 4 trials with a 20-s clicker and 4 trials with a 10 -s clicker. Each trial terminated with the delivery of a single food pellet. Trial sequences in both groups were randomized with the constraint that no more than two of the same type could occur in succession. Each trial was separated by an ITI that had a mean of $480 \mathrm{~s}$ (range $=280-740 \mathrm{~s}$ ). The ITI sequence was ordered so that Group Continuous-10/20 and Group Partial-10/5 received food at the same time within a session. The first of two extinction test sessions commenced on the day following the final session of conditioning. The first 4 trials for Group Partial-10/5 and the first 2 trials for Group Continuous-10/20 of the first extinction session proceeded as in the previous conditioning session. The remainder of this session comprised 14 nonreinforced trials with a 10-s clicker, which were repeated in the following session. The mean ITI for the trials conducted in extinction for each group was $360 \mathrm{~s}$ (range $=240-480$ s). Procedural details that have been omitted were the same as for Experiment 1.

\section{Results and Discussion}

The mean difference scores for the two groups throughout the 15 sessions of conditioning can be seen in the left panel of Figure 8 . There is an indication that conditioning was more effec-

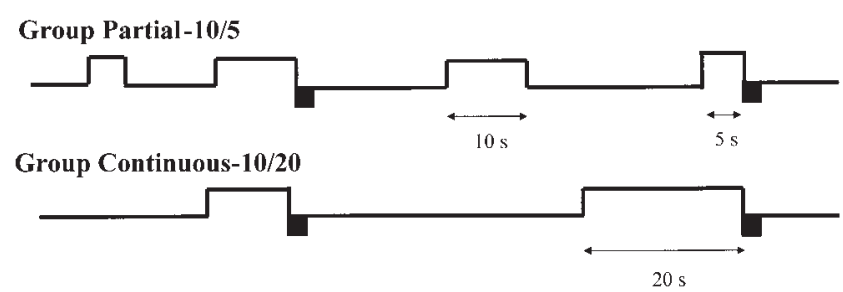

Figure 7. Procedure and design of Experiment 4. Time is indicated left to right. Open boxes represent conditioned stimulus presentations. Black boxes represent unconditioned stimulus presentations. 

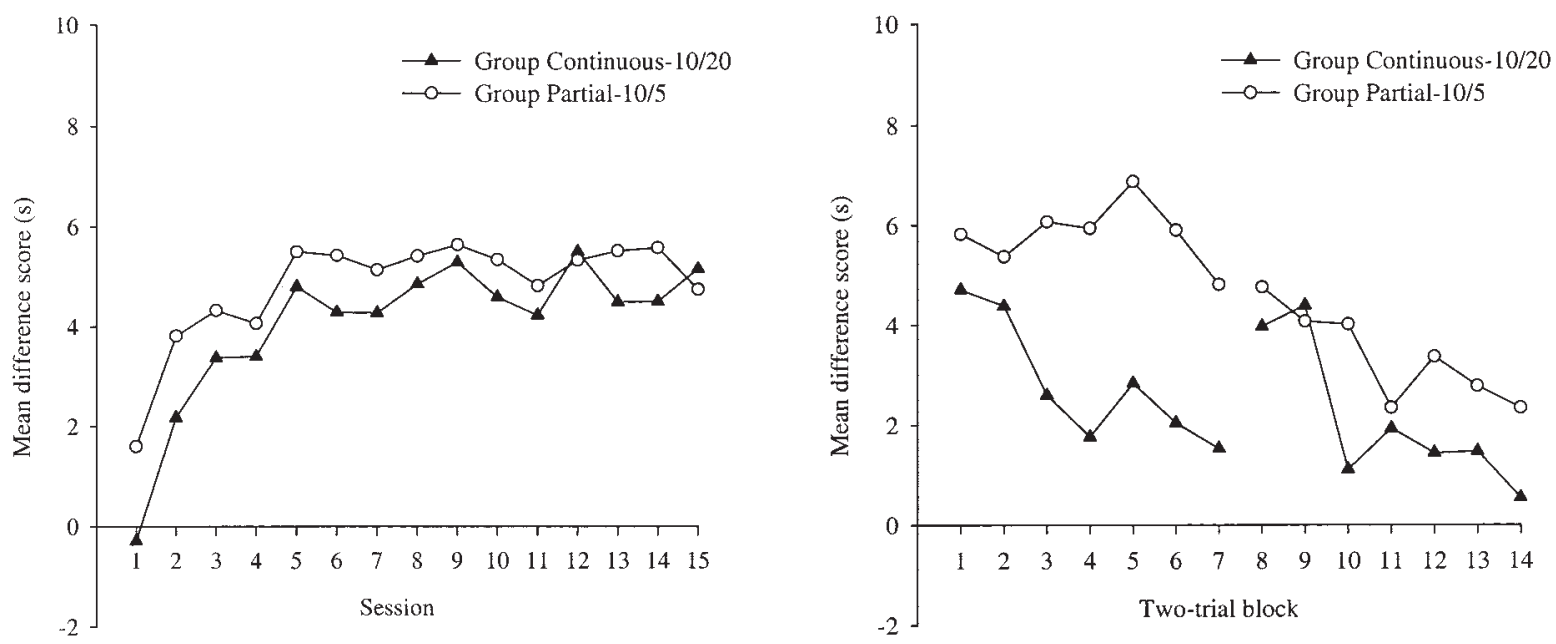

Figure 8. The mean difference scores for Groups Continuous-10/20 and Partial-10/5 across the 15 sessions of conditioning (left panel) and across the 2 sessions of extinction (right panel) of Experiment 4.

tive with Group Partial-10/5 than Group Continuous-10/20. However this observation was not supported by a two-way ANOVA of individual difference scores, which revealed a significant effect of session, $F(14,196)=8.49$, but the effect of group, $F(1,14)=$ 1.79 , and the interaction $(F<1)$, were not significant. The mean durations of magazine activity during the 10 -s periods prior to the onset of the clicker, averaged across all the sessions of conditioning, were $0.50 \mathrm{~s}$ for Group Continuous-10/20 and $0.74 \mathrm{~s}$ for Group Partial-10/5. The difference between these means was not significant, $t(14)=1.06$. It is possible that using intermixed trial durations of $10 \mathrm{~s}$ and $20 \mathrm{~s}$ in the Group Continuous-10/20 resulted in inhibition of delay that would weaken responding at the start of the CS and perhaps facilitate extinction with the 10-s CS in the test phase. Inspection of the left panel of Figure 8 shows that by the end of conditioning the performance of the two groups was very similar, which reduces concerns about the influence of inhibition of delay on the outcome of the experiment. Furthermore, the mean difference scores were calculated for the first $5 \mathrm{~s}$ of the clicker on the 10-s trials during the final session of conditioning for Group Continuous-10/20 and Group Partial-10/5. These difference scores were $2.30 \mathrm{~s}$ and $1.93 \mathrm{~s}$ for Group Continuous-10/20 and Group Partial-10/5, respectively. The difference between these means was not significant, $t(14)=0.96$. Nonetheless, to evaluate the potential influence of inhibition of delay on the course of extinction further, the latency to enter the magazine after the start of each trial during the first two and final two sessions of conditioning were recorded for both groups. The mean latencies for the first two sessions were 2.97 s for Group Continuous-10/20 and 3.16 s for Group Partial-10/5. The mean latencies for the final two sessions were 1.98 s for Group Continuous-10/20 and 1.88 s for Group Partial-10/5. A two-way ANOVA of individual latencies revealed a significant effect of session, $F(1,14)=10.83$, but no effect of group and no interaction $\left(F_{\mathrm{s}}<1\right)$. That the mean latency to enter the magazine reduced for both groups between the start and end of conditioning suggests that inhibition of delay did not develop within the CS for either group.

The group mean difference scores are shown, in two-trial blocks, for the two sessions of extinction in the right panel of
Figure 8. In both extinction sessions the strength of conditioned responding declined more rapidly in Group Continuous-10/20 than in Group Partial-10/5. A two-way ANOVA of individual difference scores revealed a significant effect of group, $F(1,14)=6.61$, of trial block, $F(13,182)=10.78$, and a significant Group $\times$ Trial Block interaction, $F(13,182)=3.99$. An analysis of simple effects revealed differences between the groups on Trial Blocks 3 to 7 and $10, F \mathrm{~s}(1,196)>7.04$. The numerical difference between the groups evident on the first two-trial block of extinction was largely a consequence of the change in performance that occurred on Trial 2. The mean difference scores on Trial 1 of the extinction stage was $5.01 \mathrm{~s}$ for Group Continuous-10/20 and 5.33 s for Group Partial-10/5. This difference between the groups was not significant, $t(14)=0.27$. The mean number of two-trial blocks that elapsed before the extinction criterion was met were 8.25 in Group Partial-10/5 and 3.00 in Group Continuous-10/20. This difference between the groups was significant, $t(14)=3.30$. The mean duration of magazine activity for the pre-CS periods averaged across all the extinction trials was $0.25 \mathrm{~s}$ for Group Continuous$10 / 20$ and 0.24 s for Group Partial-10/5. This difference was not significant, $t(14)=0.10$.

In keeping with the results from the previous three experiments, extinction progressed more rapidly in a group that received a continuous rather than a partial reinforcement schedule during conditioning. The occurrence of this effect is difficult to explain with the theory of Gallistel and Gibbon (2000) because the rate at which the US was presented during the CS was the same for both groups.

\section{General Discussion}

Four experiments compared the rates of extinction in groups that had previously received either a partial or a continuous schedule of reinforcement in Pavlovian conditioning. Three different techniques were used to equate, between groups, the rate of reinforcement during conditioning. Extinction progressed more rapidly in every experiment when conditioning had previously been conducted with a continuous rather than a partial reinforcement sched- 
ule. These results are problematic for any account of the PREE that attributes the effect to differences in the rate of reinforcement (Gallistel \& Gibbon, 2000, 2002).

The results also pose a problem for the majority of associative theories of Pavlovian conditioning, because partial reinforcement should result in weaker associative strength, and hence more rapid extinction, than continuous reinforcement. Given this shortcoming, Pearce et al. (1997) proposed that the PREE in Pavlovian conditioning might be explained in the same manner that has proved relatively successful for instrumental conditioning. That is, the nonreinforced trials during conditioning with a partial reinforcement schedule might leave an aftereffect that persists to the next trial. If this trial is reinforced, then the aftereffects of nonreinforcement will serve as a component of the context for the reinforced trial, and thereby serve as a cue for conditioned responding. The occurrence of this cue during extinction will then encourage responding to a greater extent by subjects trained with a partial rather than a continuous reinforcement schedule.

An obvious implication of this explanation is that a PREE should not be observed in Pavlovian conditioning when the effects of the different reinforcement schedules are compared using the same subjects. Provided that the trials are intermixed, conditioning with the stimuli that are consistently and intermittently paired with food will take place in the context of the aftereffects of previously nonreinforced trials. The occurrence of this context during extinction will then promote responding to the same degree for both stimuli, and the principal determinant of the rate of extinction will be their associative strengths. In support of this analysis, Pearce et al. (1997) have reported a reversed PREE in Pavlovian appetitive conditioning with rats using a within-subject methodology (see also Bouton \& Sunsay, 2001).

At least one theory of associative learning is capable of providing an account of the PREE without appealing to the influence of aftereffects of nonreinforced trials. Mackintosh (1975) proposed that the associability of a CS (that is, the ease with which its associative strength can be changed) is governed by its history of reinforcement. Of particular relevance to the present discussion is the prediction from the theory that the associability of a CS will be greater after it has been consistently rather than intermittently paired with a US. If this is correct, then the effects of extinction would be expected to be more pronounced after conditioning with a continuous than a partial reinforcement schedule. Support for this explanation of the PREE can be found in an experiment on autoshaping in pigeons by Rescorla (1999a), which demonstrated that the changes in associative strength during extinction are less after partial than continuous reinforcement. A problem with this explanation for the PREE, is that it fails to explain the reverse effect which has been reported when the same group of subjects are trained with both reinforcement schedules (Pearce et al., 1997). Perhaps, therefore, at least three different processes influence extinction after partial and continuous reinforcement: the associative strength of the CS, its associability, and the presence of the aftereffects of nonreinforcement. Given that the influence of these processes is likely to vary in different tasks, it should not be surprising if experimental tests for the PREE occasionally reveal conflicting outcomes. Thus in contrast to the reversed PREE that was reported by Pearce et al. (1997) using a within-subject methodology, Rescorla (1999b) has demonstrated a conventional PREE using pigeons and autoshaping. Perhaps Pearce et al. (1997) ob- served the opposite of a PREE because the difference between the associative strengths of the two stimuli was the dominant factor that determined the course of extinction, whereas in the study by Rescorla (1999b), it may have been the associability of the CS that was principally responsible for the outcome of the experiment.

In several of the experiments reported here, a continuous reinforcement schedule was used in which food was presented either at the midpoint or at the end of the CS. It is conceivable that animals would come to anticipate the delivery of food at both points during the CS, so that on each trial there was an occasion when an anticipated US was omitted. If the absence of the US were to generate the aftereffects that are assumed to be produced by the nonreinforced trials of a partial reinforcement schedule, then a PREE might also be expected with this schedule of reinforcement. The experiments did not include the necessary control groups to permit a satisfactory evaluation of this prediction, but the results from Experiment 1 suggest it is unlikely to be confirmed. In that experiment, both groups received a reinforcement schedule that resulted in food being unexpectedly omitted at the midpoint and the end of a 10-s CS. However, responding weakened during extinction considerably more slowly in the group that received nonreinforced trials during conditioning. On the basis of this finding it appears that it is the aftereffects of a nonreinforced trial that are critical for a demonstration of the PREE, not the unexpected omission of a US within a reinforced trial.

In contrast to the complexity of the associative analysis, the account of extinction and the PREE offered by Gallistel and Gibbon (2000) is attractively simple. According to Gallistel and Gibbon, responding ceases during extinction when the animal makes a decision to stop responding. This decision is determined by the ratio of (a) the duration of exposure to the CS since the last reinforced trial to (b) the average duration of exposure to the $\mathrm{CS}$ between successive presentations of the US during conditioning. In the current experiments, the value of this ratio was held constant between different groups of rats. Nevertheless, a reliable PREE was still observed. These results complement those of Haselgrove and Pearce (2003) who showed that extinction was facilitated by an increase or a decrease in the duration of the CS following conditioning, even though the value of this ratio was again held constant between groups. Taken together, the results from both sets of experiments suggest that the theory of Gallistel and Gibbon (2000) falls short of providing a satisfactory account of the effects of nonreinforcement in Pavlovian conditioning. We suggest that the effects of nonreinforcement are best understood from an associative perspective (e.g., Mackintosh, 1975; Pearce, 1987, 1994; Rescorla \& Wagner, 1972) that takes into account the effects that the omission of an expected US will have on the context in which it is presented (Pearce et al., 1997).

\section{References}

Amsel, A. (1958). The role of frustrative nonreward in noncontinuous reward situations. Psychological Bulletin, 55, 102-119.

Amsel, A. (1992). Frustration theory. Cambridge, England: Cambridge University Press.

Bouton, M. E., \& Sunsay, C. (2001). Contextual control of appetitive conditioning: Influence of a contextual stimulus generated by a partial reinforcement procedure. Quarterly Journal of Experimental Psychology: Comparative and Physiological Psychology, 54B, 109-125.

Capaldi, E. J. (1967). A sequential theory of instrumental training. In K. W. 
Spence \& J. T. Spence (Eds.), The psychology of learning and motivation (pp. 67-156). New York: Academic Press.

Carew, T. J., Pinsker, H. M., \& Kandel, E. R. (1972, January 28). Longterm habituation of a defensive withdrawal reflex in aplysia. Science, $175,451-454$.

Fitzgerald, R. D. (1963). Effects of partial reinforcement with acid on the classically conditioned salivary response in dogs. Journal of Comparative and Physiological Psychology, 56, 1056-1060.

Fitzgerald, R. D., Vardaris, R. M., \& Teyler, T. J. (1966). Effects of partial reinforcement followed by continuous reinforcement on classically conditioned heart-rate in the dog. Journal of Comparative and Physiological Psychology, 62, 483-486.

Gallistel, C. R., \& Gibbon, J. (2000). Time, rate, and conditioning. Psychological Review, 107, 289-344.

Gallistel, C. R., \& Gibbon, J. (2002). The symbolic foundations of conditioned behavior. Mahwah, NJ: Erlbaum.

Gibbon, J., Farrell, L., Locurto, C. M., Duncan, H. J., \& Terrace, H. S. (1980). Partial reinforcement in autoshaping with pigeons. Animal Learning and Behavior, 8, 45-59.

Gibbs, C. M., Latham, S. B., \& Gormezano, I. (1978). Classical conditioning of the rabbit nictitating membrane response: Effects of reinforcement schedule on response maintenance and resistance to extinction. Animal Learning and Behavior, 6, 209-215.

Guthrie, E. R. (1935). The psychology of learning. New York: Harper.

Haselgrove, M., \& Pearce, J. M. (2003). Facilitation of extinction by an increase or a decrease in trial duration. Journal of Experimental Psychology: Animal Behavior Processes, 29, 153-166.

Hull, C. L. (1943). Principles of behaviour. New York: Appleton-CenturyCrofts.

Kimble, G. A. (1961). Hilgard and Marquis' conditioning and learning. New York: Appleton-Century-Crofts.

Kirk, R. E. (1968). Experimental design: Procedures for the behavioural sciences. Belmont, CA: Brooks/Cole.

Mackintosh, N. J. (1974). The psychology of animal learning. London: Academic Press.

Mackintosh, N. J. (1975). A theory of attention: Variations in the associability of stimuli with reinforcement. Psychological Review, 82, 276298

Pavlov, I. P. (1927). Conditioned reflexes. New York: Dover.
Pearce, J. M. (1987). A model for stimulus generalization in Pavlovian conditioning. Psychological Review, 94, 61-73.

Pearce, J. M. (1994). Similarity and discrimination: A selective review and a connectionist model. Psychological Review, 94, 587-607.

Pearce, J. M., Redhead, E. S., \& Aydin, A. (1997). Partial reinforcement in appetitive Pavlovian conditioning with rats. Quarterly Journal of Experimental Psychology, 50B, 274-294.

Pfautz, P. L. (1980). Unconditioned facilitation and diminution of the unconditioned response. Unpublished doctoral thesis, Yale University.

Polin, A. T. (1959). The effects of flooding and physical suppression as extinction techniques on an anxiety motivated avoidance locomotor response. Journal of Psychology, 47, 235-245.

Rescorla, R. A. (1999a). Partial reinforcement reduces the associative change produced by nonreinforcement. Journal of Experimental Psychology: Animal Behavior Processes, 25, 403-414.

Rescorla, R. A. (1999b). Within-subject partial reinforcement extinction effect in autoshaping. Quarterly Journal of Experimental Psychology, 52B, 75-87.

Rescorla, R. A., \& Wagner, A. R. (1972). A theory of Pavlovian conditioning: Variations in the effectiveness of reinforcement and nonreinforcement. In A. H. Black \& W. F. Prokasy (Eds.), Classical conditioning II (pp. 64-99). New York: Appleton-Century-Crofts.

Slivka, R. M., \& Bitterman, M. E. (1966). Classical appetitive conditioning in the pigeon: Partial reinforcement. Psychonomic Science, 4, 181-182.

Teichner, W. H. (1952). Experimental extinction as function of the intertrial intervals during conditioning and extinction. Journal of Experimental Psychology, 44, 170-178

Terry, W. S. (1976). Effects of priming unconditioned stimulus representation in short-term memory on Pavlovian conditioning. Journal of Experimental Psychology: Animal Behavior Processes, 2, 354-369.

Thorndike, E. L. (1911). Animal intelligence. New York: Macmillan.

Wagner, A. R., Siegel, S., Thomas, E., \& Ellison, G. D. (1964). Reinforcement history and the extinction of a conditioned salivary response. Journal of Comparative and Physiological Psychology, 58, 354-358.

Received December 22, 2003

Revision received March 12, 2004 Accepted March 12, 2004 